\title{
Criterios de evaluación y utilización de revistas académicas en el área de conocimiento de Psicología en España
}

\author{
José Antonio SALVADOR OLIVÁN \\ Universidad de Zaragoza \\ jaso@unizar.es \\ Rosario ARQUERO AVILÉS \\ Universidad Complutense de Madrid \\ carquero@ccinf.ucm.es \\ Genaro LAMARCA LANGA \\ Universidad de Zaragoza \\ glamarca@unizar.es
}

Recibido: 31/03/2011

Aceptado: 4/04/2011

\section{RESUMEN}

Análisis de los resultados de una encuesta enviada a los profesores e investigadores de Psicología de las universidades españolas y del Consejo Superior de Investigaciones Científicas. Se estudian los criterios de selección de artículos para su lectura, los puntos de vista sobre la forma de seleccionar los artículos para su publicación por las revistas y las opiniones sobre la evaluación de la calidad de artículos y de revistas. El principal resultado del estudio es un ranking de revistas españolas y extranjeras basado en el uso y valoración de las mismas y una distribución de dichas revistas por áreas específicas de conocimiento en Psicología.

Palabras clave: Evaluación de revistas científicas; criterios de selección de artículos; Ciencias Sociales; Bibliometría; Psicología.

Criteria for assessment and use of academic journals in Psychology Area in Spain

\begin{abstract}
Analysis of results of a survey sent to teachers and researchers of Psychology of universities and Council for Scientific Research in Spain. We study criteria for selecting articles for reading, their viewpoints on how to select articles for publication in journals and their views about the quality assessment of articles and journals. The main result of this study is a ranking of Spanish and foreign journals based on the use and assessment of their quality and distribution of these journals by specific areas of knowledge in Psychology.

Key words: Quality assessment of scientific journals; criteria for articles selection; Social Sciences; Bibliometrics; Psychology.
\end{abstract}




\section{INTRODUCCIÓN}

Debido al proceso de adaptación al Espacio Europeo de Educación Superior (EEES), la evaluación de la calidad de las universidades ha cobrado especial protagonismo en los últimos años en España (Buela-Casal, 2005). En este contexto y, como parte fundamental de la evaluación del profesorado universitario, se sitúa la evaluación de la investigación, pilar básico en el que se sustenta la política científica en términos de concesión de incentivos y distribución de fondos para la obtención de financiación.

La investigación científica, como una parte de la actividad académica, se manifiesta en diferentes resultados como la dirección de trabajos de investigación, la participación en proyectos de investigación, las tareas de dirección y gestión de publicaciones científicas o la producción de publicaciones científicas.

En el ámbito de las Ciencias Sociales se han publicado numerosos trabajos sobre evaluación de la investigación en dicho ámbito en el panorama internacional (Baker, 1992; Bush, Epstein y Sainz, 1997; Cheung, 1990; Cnaan, Caputo y Shmuely, 1994; Furr, 1995; Nelson, Buss y Katzko, 1983, entre otros) y en disciplinas específicas de dicho ámbito como la Sociología (Glenn, 1971; Lindsey and Kirk, 1992; Teevan, 1980), las Ciencias Políticas (Garand, 1990; Gilles, Mizell y Patterson, 1989; Lester, 1990; Nisonger, 1993), las Ciencias de la Educación (Council on Social Work Education, 2002; Reamer, 1992; West, Carmody y Stallings, 1983), la Economía (Alexander y Mabry, 1994; Brinn, Jones y Pendlebury, 2001; Chan, Fok y Pan, 2000; Easton y Easton, 2003; Franke, Edlund, y Oster, 1990; Fry, Walters y Scheuermann, 1985; Geary, Marriott y Rowlinson, 2004; Otley, 2002; Parker, Guthrie y Gray, 1998; Zeff, 1996, entre otros) o la Psicología (Boor, 1973; Dorelan, 1989; Gynther, 1973; Mace y Warner, 1973).

En concreto, la evaluación de la investigación basada en la valoración de las publicaciones (y específicamente de revistas científicas) se ha convertido en el objetivo de multitud de trabajos en los que sustentar dicha evaluación. En este sentido, y según señalan Alcaín y Román (2005a), los primeros estudios de evaluación de publicaciones periódicas en el panorama internacional datan de finales de los cincuenta del siglo XX, aunque hasta finales de los setenta no empiezan a realizarse en España estudios sobre la calidad de las revistas científicas y técnicas. Estos estudios se centran inicialmente en el ámbito de las disciplinas científico-tecnológicas y, progresivamente, en el ámbito de las disciplinas de Ciencias Sociales y Humanas en el que, debido a la profusión de trabajos en nuestro país en los últimos años, destacan dos disciplinas concretas: la Economía y la Psicología.

Entre las aportaciones que presentan propuestas de análisis y evaluación de revistas científicas, que han surgido en el contexto específico de la evaluación de la investigación en Psicología en España y cuya responsabilidad es de autores de dicha área de conocimiento, cabe citar los siguientes: Agudelo, Bretón-López y Buela-Casal (2003); Alcaín Partearroyo y Román Román (2005a y b); Alcaín Partearroyo y RuizGálvez (2001); Buela-Casal (2003); Buela-Casal, Carretero-Dios y De los Santos-Roig (2002); Carpintero, Gotor y Miralles (1983a y b); Carpintero y Tortosa (1996); 
Carretero-Dios, De los Santos Roig y Buela-Casal (2005); Gotor y Miralles (1985); Peñaranda Ortega, Quiñones Vidal y López García (2005); Tortosa Gil y Civera Moyá (2001), entre otros.

Por nuestra parte, hemos de señalar que en este trabajo presentamos los resultados de un estudio evaluativo de revistas científicas del área de Psicología que se inserta en un proyecto de evaluación de la calidad de las revistas científicas españolas en Humanidades y Ciencias Sociales ${ }^{1}$, sobre cuyo alcance y resultados específicos pueden consultarse otras publicaciones previas ${ }^{2}$. El fin de este trabajo es conseguir que nuestra investigación constituya una aportación complementaria a trabajos previos de evaluación de revistas científicas de Psicología en España.

\section{OBJETIVOS}

Los objetivos específicos de este trabajo son:

1. Identificar los criterios más utilizados en la selección de artículos para su lectura por parte de profesores e investigadores pertenecientes al área de Psicología.

2. Medir la percepción sobre algunos aspectos importantes relacionados con la evaluación y calidad de las revistas en Psicología.

3. Identificar las revistas más utilizadas en las diversas áreas de conocimiento de Psicología.

\section{MATERIAL Y MÉTODO}

Se describe a continuación el método utilizado en el proyecto de investigación ERCE de evaluación de la calidad de las revistas científicas españolas en Humanidades y Ciencias Sociales, al que nos hemos referido, aunque para este trabajo sólo se seleccionaron a los profesores e investigadores pertenecientes al área de Psicología.

Población de estudio y marco muestral: La población sujeta a estudio estuvo formada por todo el personal docente e investigador de las universidades españolas y de los centros $\mathrm{u}$ organismos adscritos al CSIC (Consejo Superior de Investigaciones Científicas) perteneciente a las áreas de conocimiento de Ciencias Sociales y Humanidades.

La constitución del marco se pensó que debería estar formada por la lista de direcciones de correos electrónicos de toda la población. Para conseguir esa información se envió una carta a los rectores, vicerrectores de investigación o gerentes de las universidades y centros de investigación españoles solicitando el listado del

${ }^{1}$ Proyecto de investigación ERCE (Evaluación de la calidad de las revistas científicas españolas en Humanidades y Ciencias Sociales), financiado por el Ministerio de Ciencia y Tecnología (BFF200308313).

${ }^{2}$ GRUPO DE INVESTIGACIÓN ERCE (2007); Lamarca et al., (2005), Salvador, Lamarca y Arquero (2009a), Salvador, Lamarca y Arquero (2009b). 
personal de cada universidad. La respuesta a esta petición fue muy variada, desde aquellos que enviaron el listado completo hasta las que no dieron respuesta alguna, pasando por aquellas que contestaron que ellos se encargarían de distribuir la carta en la que se solicitaba la colaboración del profesor/investigador, otras que remitían a las direcciones que aparecían en sus páginas web o algunas que sugerían ponernos en contacto con los propios departamentos. Transcurrido un mes, se volvió a enviar otra carta a las universidades de las que no se obtuvo ninguna respuesta. Finalmente, de aquellas que siguieron sin dar ninguna respuesta, se realizó una búsqueda en sus sitios web con el objetivo de conseguir el mayor número de direcciones posible.

Se desconoce el número total exacto de personal al que se le envió un correo electrónico invitándole a participar en la encuesta, ya que hubo universidades que se comprometieron a hacerlo ellas mismas o a través de sus propios departamentos y no informaron de cuántos habían enviado. El número de correos personales que se enviaron por parte del personal del Proyecto fue de 20542, a los que hay que sumar los correos enviados por 11 universidades a su propio personal.

Contacto con los participantes y seguimiento: La invitación a participar en el estudio se envió por correo electrónico a todos los individuos que formaban parte del marco muestral. El mensaje explicaba el propósito del estudio y solicitaba la participación de cada individuo, proporcionando un enlace a la dirección web en la que estaba al cuestionario online. Al no saber con exactitud el número de correos que se enviaron tampoco se puede conocer la tasa de respuesta.

Posteriormente, se volvió a enviar otro correo global como recordatorio y, después, otro solamente a aquellos profesores que pertenecían a áreas de conocimiento en las que se había obtenido un número de respuestas inferior a 8.

Los datos se recogieron a través de un cuestionario web interactivo. Posteriormente, se analizó la validez de las encuestas, eliminando aquellas duplicadas o que estaban totalmente en blanco, quedando al final 2398 encuestas válidas para los propósitos del estudio.

Áreas de conocimiento: Se seleccionaron profesores e investigadores de todas las áreas de conocimiento pertenecientes a Ciencias Sociales y Humanidades. Para su posterior análisis, se agruparon en 5 grandes macro áreas: Humanidades, Económicas y Empresariales, Derecho, Sociales y Educación.

Contenido del cuestionario: El instrumento utilizado para recoger los datos fue una encuesta web, diseñada conjuntamente con el personal del Centro de Documentación Científica de la Universidad de Zaragoza.

El cuestionario constaba de cuatro partes. La primera de ellas recogía datos demográficos entre los que se incluían el cargo académico o categoría profesional, el área de conocimiento específica y la universidad o centro de investigación a la que pertenecían. Las dos últimas partes estaban relacionadas con la evaluación de las revistas españolas y extranjeras.

La segunda sección, que es la que se analiza en este trabajo, tenía como objetivo conocer la opinión de los encuestados sobre los criterios utilizados en la selección de un artículo para su lectura: 

a) Autor
b) Tema de que trata
c) La revista en la que se publica
d) La fecha de publicación, y
e) Si es citado en otra publicación

Y otros aspectos relacionados con la evaluación de la investigación:

a) El medio más adecuado para evaluar la investigación de un autor es asignarle el resultado de la evaluación de las revistas donde ha publicado,

b) La publicación de un artículo en una revista extranjera es garantía de mayor calidad que su publicación en una revista española,

c) El sistema de selección de artículos más adecuado para su publicación en una revista es la revisión anónima por expertos, y

d) La calidad de una revista depende del número de citas que reciben sus artículos por otras publicaciones.

Las variables se midieron utilizando una escala ordinal codificadas de la siguiente manera:

$1=$ En total desacuerdo,

$2=$ Bastante en desacuerdo,

$3=$ Bastante de acuerdo, $y$

4= Completamente de acuerdo.

Análisis estadístico: Se ha utilizado el programa SPSS14.0 para realizar la estadística. Los análisis incluyen la estadística descriptiva, el cálculo de los intervalos de confianza, test de chi cuadrado y coeficiente de contingencia como test de independencia, el test de Friedman para contrastar diferencias entre los grupos sometidos a comparación, y el coeficiente de concordancia W de Kendall para comprobar el grado de concordancia ante las diferentes preguntas realizadas.

\section{LIMITACIONES DEL ESTUDIO}

Las encuestas de opinión a través de la web presentan una serie de limitaciones debidas a tasas de respuesta bajas y a que se desconoce si las muestras son representativas de la población sometida a estudio. Al no conocer el número de profesores e investigadores de cada universidad en el área de conocimiento sujeta a estudio, no podemos calcular la tasa de respuesta; en la literatura se ha documentado una tasa de respuesta baja (entre un $4 \%$ y un 14\%) en las encuestas web, si bien pueden variar según los procedimientos de seguimiento y motivación utilizados (Hayslett, 2004). 
Somos conscientes de que no se puede medir el error de muestreo y los resultados no se pueden generalizar a toda la población de profesores e investigadores de Psicología de España, pero sí son orientativos y nos van a permitir generar una serie de hipótesis, que tal y como señalan Miller y col., (1996), es una de las utilidades de los estudios exploratorios con encuestas web. Los resultados que se presentan y analizan en la siguiente sección reflejan la visión y percepción de una muy amplia muestra de profesores e investigadores universitarios en el área de conocimiento de Psicología.

\section{RESULTADOS}

El número total de encuestas válidas en Psicología es de 176, de las que 109 $(61,9 \%)$ han sido cumplimentadas por hombres y $67(38,1 \%)$ por mujeres. Algo más de la mitad (54,5\%) pertenecen al cuerpo de profesores titulares de universidad, y el menor porcentaje a titulares y catedráticos de escuela universitaria.

La distribución por áreas de conocimiento revela que el 27,3\% de los encuestados pertenece a Psicología Evolutiva y de la Educación, seguida de Personalidad, Evaluación y Tratamiento Psicológico con un 23,3\%, siendo Psicobiología con un 4,5\% la menos representada.

Los encuestados proceden de 34 universidades españolas, si bien la mitad se concentran sólo en 6 universidades, siendo la de Valencia la que mayor número de respuestas presentó. Algo menos de la mitad $(42,0 \%)$ no tenían reconocido ningún tramo de investigación, y la tercera parte tenían al menos 2 sexenios.

Tabla I-Características generales y socio-demográficas de la muestra de profesores e investigadores de Psicología

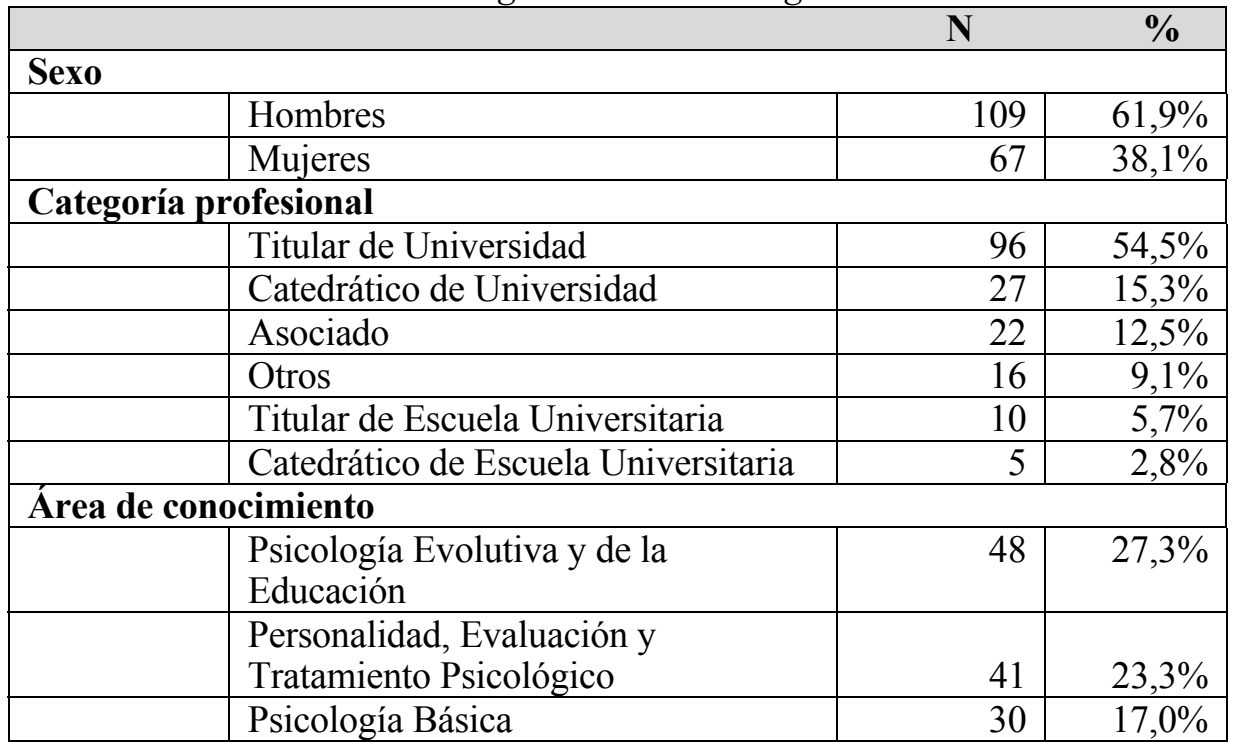




\begin{tabular}{|l|l|r|r|}
\hline & Psicología Social & 30 & $17,0 \%$ \\
\hline & $\begin{array}{l}\text { Metodología de las Ciencias del } \\
\text { Comportamiento }\end{array}$ & 19 & $10,8 \%$ \\
& Psicobiología & 8 & $4,5 \%$ \\
\hline & \multicolumn{3}{|l|}{} \\
\hline Universidad de procedencia & 32 & $18,2 \%$ \\
\hline & Valencia & 13 & $7,4 \%$ \\
\hline & Oviedo & 12 & $6,8 \%$ \\
\hline & Complutense de Madrid & 12 & $6,8 \%$ \\
\hline & Granada & 12 & $6,8 \%$ \\
\hline & Islas Baleares & 10 & $5,7 \%$ \\
\hline & La Laguna & 8 & $4,5 \%$ \\
\hline & Murcia & 8 & $4,5 \%$ \\
\hline & Pais Vasco & 7 & $4,0 \%$ \\
\hline & Santiago de Compostela & 62 & $35,2 \%$ \\
\hline & Otras & 74 & $42,0 \%$ \\
\hline Sexenios & Ninguno & 41 & $23,3 \%$ \\
\hline & 1 sexenio & 61 & $34,7 \%$ \\
\hline & Más de 1 sexenio & \multicolumn{3}{|l}{} \\
\hline
\end{tabular}

\subsection{Criterios utilizados en la selección de un artículo para su lectura}

La tabla II muestra el porcentaje de encuestados que están de acuerdo o completamente de acuerdo con cada uno de los criterios utilizados en la selección de un artículo para su lectura. Lógicamente, la gran mayoría de los encuestados seleccionan los artículos por el tema tratado en el artículo, siendo el medio o la revista donde se publica el segundo criterio con mayor porcentaje de respuestas favorables. El hecho de ser citado en otra publicación es el criterio menos valorado.

Existe un grado de acuerdo importante en la valoración de estos criterios, destacando positivamente que el tema cubierto en el artículo y la revista donde se publica son más importantes que el autor, el ser citado en otra publicación o la fecha. 
Tabla II- Criterios utilizados en la selección de un artículo para su lectura:

Distribución de respuestas de acuerdo/completamente de acuerdo y rango medio de la puntuación.

\begin{tabular}{|l|rr|r|}
\hline & \multicolumn{1}{|c|}{ \% $^{\mathbf{a}}$} & \multicolumn{1}{c|}{ I.C. } & Rango medio $^{\mathbf{b}}$ \\
\hline Autor & $51,7 \%$ & $44,3 \%-59,1 \%$ & 2,62 \\
Tema o asunto & $98,9 \%$ & $97,3 \%-100 \%$ & 4,53 \\
Revista donde se publica & $64,0 \%$ & $56,9 \%-71,1 \%$ & 2,84 \\
Fecha de publicación & $55,1 \%$ & $47,8 \%-62,5 \%$ & 2,56 \\
Ser citado en otra publicación & $49,1 \%$ & $41,7 \%-56,6 \%$ & 2,45 \\
\hline
\end{tabular}

${ }^{a}$ Test de Chi cuadrado $\mathrm{p}<0,05$

${ }^{\mathrm{b}}$ Test de Friedman, $\mathrm{p}<0,05$. Coeficiente W de Kendall, 0,364.

Al analizar la valoración de estos criterios por categoría profesional (Tabla III) podemos destacar los siguientes aspectos:

- Los catedráticos de universidad conceden más importancia al autor de un artículo que el resto de las categorías profesionales.

- En cuanto a la fecha de publicación, son los catedráticos de universidad los que menos importancia le dan.

- En cuanto al asunto, revista y ser citado en otra publicación, no existen diferencias significativas entre las diferentes categorías profesionales.

Tabla III- Porcentaje de respuestas favorables (De acuerdo/Completamente de acuerdo) en los criterios utilizados para la selección de un artículo para su lectura según categoría profesional. (Porcentaje, Residuos tipificados corregidos)

\begin{tabular}{|c|c|c|c|c|c|}
\hline & Autor $^{a}$ & Asunto & Revista & $\begin{array}{l}\text { Fecha } \\
\text { publicación }^{\text {a }}\end{array}$ & $\begin{array}{l}\text { Citado en } \\
\text { otra } \\
\text { publicación }\end{array}$ \\
\hline Catedrático de Universidad & $\begin{array}{r}74,1 \% \\
(2,5)\end{array}$ & $96,3 \%$ & $59,3 \%$ & $\begin{array}{r}29,6 \% \\
(-2,9)\end{array}$ & $53,8 \%$ \\
\hline Titular de Universidad & $\begin{array}{r}47,9 \% \\
(-1,1)\end{array}$ & $99,0 \%$ & $66,7 \%$ & $\begin{array}{r}56,3 \% \\
(0,3)\end{array}$ & $45,7 \%$ \\
\hline $\begin{array}{l}\text { Catedrático/Titular Escuela } \\
\text { Universitaria }\end{array}$ & $\begin{array}{r}40,0 \% \\
(-0,9)\end{array}$ & $100,0 \%$ & $64,3 \%$ & $\begin{array}{r}66,7 \% \\
(0,9)\end{array}$ & $60,0 \%$ \\
\hline Asociado & $\begin{array}{r}36,4 \% \\
(-1,5)\end{array}$ & $100,0 \%$ & $68,2 \%$ & $\begin{array}{r}72,7 \% \\
(1,8)\end{array}$ & $50,0 \%$ \\
\hline Otros & $\begin{array}{r}68,8 \% \\
(1,4)\end{array}$ & $100,0 \%$ & $50,0 \%$ & $\begin{array}{r}56,3 \% \\
(0,1)\end{array}$ & $50,0 \%$ \\
\hline
\end{tabular}

${ }^{a}$ Test de Chi cuadrado $\mathrm{p}<0,05$. Coeficiente de contingencia, $\mathrm{p}<0,05$

La valoración de estos criterios según el área de conocimiento al que pertenecen los encuestados revela lo siguiente (Tabla IV): 
- Ningún profesor del área de Psicobiología da importancia a la autoría de un artículo para su selección.

- En todas las áreas de conocimiento hay una coincidencia plena en que el tema tratado en un artículo es un criterio esencial.

- No existen diferencias de valoración y opinión entre la distintas áreas en relación con los criterios de la revista, fecha de publicación o el hecho de ser citado en otra publicación.

Tabla IV-Porcentaje de respuestas favorables (De acuerdo/Completamente de acuerdo) en los criterios utilizados para la selección de un artículo para su lectura según área de conocimiento. (Porcentaje, Residuos tipificados corregidos)

\begin{tabular}{|c|c|c|c|c|c|}
\hline & Autor $^{a}$ & Asunto & Revista & $\begin{array}{l}\text { Fecha } \\
\text { publicación }\end{array}$ & $\begin{array}{l}\text { Citado en } \\
\text { otra } \\
\text { publicación }\end{array}$ \\
\hline $\begin{array}{l}\text { Metodología de las Ciencias del } \\
\text { Comportamiento }\end{array}$ & $\begin{array}{r}47,4 \% \\
(-0,4)\end{array}$ & $\begin{array}{r}100,0 \\
\%\end{array}$ & $78,9 \%$ & $42,1 \%$ & $66,7 \%$ \\
\hline $\begin{array}{l}\text { Personalidad, Evaluación y } \\
\text { Tratamiento Psicológico }\end{array}$ & $\begin{array}{r}48,8 \% \\
(-0,4)\end{array}$ & $\begin{array}{r}100,0 \\
\%\end{array}$ & $61,0 \%$ & $48,8 \%$ & $58,5 \%$ \\
\hline Psicobiología & $\begin{array}{r}0 \% \\
(-3,0)\end{array}$ & $\begin{array}{r}100,0 \\
\%\end{array}$ & $100,0 \%$ & $50,0 \%$ & $50,0 \%$ \\
\hline Psicología Básica & $\begin{array}{r}66,7 \% \\
(1,8)\end{array}$ & $\begin{array}{r}100,0 \\
\%\end{array}$ & $60,0 \%$ & $50,0 \%$ & $33,3 \%$ \\
\hline $\begin{array}{l}\text { Psicología Evolutiva y de la } \\
\text { Educación }\end{array}$ & $\begin{array}{r}58,3 \% \\
(1,1)\end{array}$ & $95,8 \%$ & $63,8 \%$ & $68,8 \%$ & $44,7 \%$ \\
\hline Psicología Social & $\begin{array}{r}50,5 \% \\
(-1,6)\end{array}$ & $\begin{array}{r}100,0 \\
\%\end{array}$ & $53,3 \%$ & $56,7 \%$ & $48,3 \%$ \\
\hline
\end{tabular}

${ }^{a}$ Test de Chi cuadrado $\mathrm{p}<0,05$. Coeficiente de contingencia, $\mathrm{p}<0,05$

\subsection{Grado de acuerdo con determinados aspectos relacionados con las publicaciones}

El 96,6\% de los encuestados están de acuerdo con que la revisión anónima por expertos es el procedimiento más adecuado para la selección de artículos y su publicación en revistas. Destaca que algo menos de la tercera parte $(30,9 \%)$ consideran que publicar en una revista extranjera sea garantía de mayor calidad que en una revista española, obteniendo este criterio una puntuación significativamente más baja (rango medio 1,77) que el resto de los ítems evaluados.

Los dos enunciados restantes obtienen una puntuación de aprobado, como así indica el rango medio y el porcentaje de respuestas favorables, donde aproximadamente sólo la mitad están de acuerdo con que el medio más adecuado para evaluar la investigación de un autor es evaluar las revistas donde publica y con que la calidad de una revista depende del número de citas recibidas, criterios en los que se basa fundamentalmente la 
evaluación de la CNEAI a los profesores e investigadores para obtener sus tramos de investigación.

Tabla V-Distribución de respuestas De acuerdo/Completamente de acuerdo relacionadas con las siguientes afirmaciones sobre la publicación de artículos y revistas (I.C. Intervalos de confianza al 95\%)

\begin{tabular}{|c|c|c|c|}
\hline & $\%^{a}$ & & $\begin{array}{l}\text { Rango } \\
\text { mediob }\end{array}$ \\
\hline $\begin{array}{l}\text { El medio más adecuado para evaluar la } \\
\text { investigación de un autor es asignarle el } \\
\text { resultado de la evaluación de las revistas } \\
\text { donde ha publicado }\end{array}$ & $52,0 \%$ & $\begin{array}{r}44,6 \%- \\
59,4 \%\end{array}$ & 2,33 \\
\hline $\begin{array}{l}\text { La publicación de un artículo en una } \\
\text { revista extranjera es garantía de mayor } \\
\text { calidad que su publicación en una revista } \\
\text { española }\end{array}$ & $30,9 \%$ & $\begin{array}{r}24,0 \%- \\
37,7 \%\end{array}$ & 1,77 \\
\hline $\begin{array}{l}\text { El sistema de selección de artículos más } \\
\text { adecuado para su publicación en una } \\
\text { revista es la revisión anónima por } \\
\text { expertos }\end{array}$ & $96,6 \%$ & $\begin{array}{r}93,9 \%- \\
99,3 \%\end{array}$ & 3,65 \\
\hline $\begin{array}{l}\text { La calidad de una revista depende del } \\
\text { número de citas que reciben sus artículos } \\
\text { por otras publicaciones }\end{array}$ & $48,0 \%$ & $\begin{array}{r}40,6 \%- \\
55,4 \%\end{array}$ & 2,25 \\
\hline
\end{tabular}

${ }^{\mathrm{a}}$ Test de Chi cuadrado $\mathrm{p}<0,05$

${ }^{\mathrm{b}}$ Test de Friedman, $\mathrm{p}<0,05$. Coeficiente W de Kendall, 0,484.

En la Tabla VI se presentan los resultados conseguidos en las categorías profesionales, destacando lo siguiente:

- Los profesores asociados y titulares o catedráticos de escuela universitaria son los que menos de acuerdo están con que la evaluación de las revistas es el medio más adecuado para evaluar la investigación de un autor.

- No se aprecian diferencias significativas entre los diferentes cuerpos de profesorado en cuanto a que la publicación de un artículo en una revista extranjera indique mayor calidad que su publicación en una revista española ni en que la revisión por expertos es el sistema de selección de artículos más adecuado para su publicación.

- Respecto a si la calidad de una revista depende del número de citas recibidas, son los catedráticos y titulares de escuela universitaria los que más en desacuerdo están con esta afirmación. 
Tabla VI- Distribución de respuestas De acuerdo/Completamente de acuerdo relacionadas con publicación de artículos y revistas según categoría profesional (Porcentajes y residuos tipificados corregidos)

\begin{tabular}{|c|c|c|c|c|}
\hline & $\begin{array}{l}\text { Evaluación } \\
\text { revistas } \\
\text { publicado }^{\text {a }}\end{array}$ & $\begin{array}{l}\text { Publicación } \\
\text { en revista } \\
\text { extranjera } \\
\end{array}$ & $\begin{array}{l}\text { Revisión } \\
\text { anónima por } \\
\text { expertos }\end{array}$ & $\begin{array}{l}\text { Calidad } \\
\text { depende del } \\
n^{0} \text { de citas }{ }^{\text {a }} \\
\end{array}$ \\
\hline Catedrático de Universidad & $\begin{array}{r}63,0 \% \\
(1,2)\end{array}$ & $25,9 \%$ & $96,3 \%$ & $\begin{array}{r}51,9 \% \\
(0,4)\end{array}$ \\
\hline Titular de Universidad & $\begin{array}{r}57,3 \% \\
(1,5)\end{array}$ & $36.5 \%$ & $97,9 \%$ & $\begin{array}{r}47,9 \% \\
(0)\end{array}$ \\
\hline $\begin{array}{l}\text { Catedrático/Titular Escuela } \\
\text { Universitaria }\end{array}$ & $\begin{array}{r}26,7 \% \\
(-2,1)\end{array}$ & $20,0 \%$ & $100,0 \%$ & $\begin{array}{r}13,3 \% \\
(-2,8)\end{array}$ \\
\hline Asociado & $\begin{array}{r}31,8 \% \\
(-2,0)\end{array}$ & $13,6 \%$ & $86,4 \%$ & $\begin{array}{r}59,1 \% \\
(1,1)\end{array}$ \\
\hline Otros & $\begin{array}{r}53,3 \% \\
(0,1)\end{array}$ & $40,0 \%$ & $100,0 \%$ & $\begin{array}{r}60,0 \% \\
(1,0)\end{array}$ \\
\hline
\end{tabular}

${ }^{a}$ Test de Chi cuadrado $p<0,05$. Coeficiente de contingencia, $p<0,05$

Del análisis de los resultados obtenidos por áreas de conocimiento, podemos destacar que:

- En todas las áreas de conocimiento la gran mayoría de profesores e investigadores están de acuerdo con que la revisión anónima por expertos es el sistema más adecuado para publicar artículos en una revista.

- Tampoco hay diferencia entre las distintas áreas en cuanto a que la publicación de un artículo en revista extranjera sea indicio de mayor calidad, obteniendo una puntuación baja en todas ellas.

- Existe una diferencia significativa en la opinión del resto de enunciados, siendo los profesores del área de Metodología de las Ciencias del Comportamiento los que están más de acuerdo con que el medio más adecuado para evaluar la investigación es evaluar las revistas donde se publica $(78,9 \%)$, frente a los que pertenecen a Psicología Evolutiva y de la Educación, donde se obtiene el menor porcentaje de respuestas favorables $(37,5 \%)$. En cuanto a si la calidad de una revista depende del número de citas que recibe, aquellos que pertenecen a las áreas de Metodología de las Ciencias del Comportamiento y Psicobiología están más de acuerdo con dicha afirmación que el resto de las áreas. 
Tabla VII- Distribución de respuestas De acuerdo/Completamente de acuerdo relacionadas con publicación de artículos y revistas según áreas de conocimiento (Porcentajes y residuos tipificados corregidos)

\begin{tabular}{|c|c|c|c|c|}
\hline & $\begin{array}{l}\text { Evaluación } \\
\text { revistas } \\
\text { publicado }^{a}\end{array}$ & $\begin{array}{l}\text { Publicación } \\
\text { en revista } \\
\text { extranjera }\end{array}$ & $\begin{array}{l}\text { Revisión } \\
\text { anónima por } \\
\text { expertos }\end{array}$ & $\begin{array}{l}\text { Calidad } \\
\text { depende del } \\
n^{0} \text { de citas }{ }^{\mathrm{a}}\end{array}$ \\
\hline Metodología de las Ciencias del & $78,9 \%$ & $42,1 \%$ & $100,0 \%$ & $78,9 \%$ \\
\hline Comportamiento & $(2,5)$ & & & $(2,9)$ \\
\hline $\begin{array}{l}\text { Personalidad, Evaluación y } \\
\text { Tratamiento Psicológico }\end{array}$ & $\begin{array}{r}46,3 \% \\
(-0,8)\end{array}$ & $34,1 \%$ & $100,0 \%$ & $\begin{array}{r}56,1 \% \\
(1,2)\end{array}$ \\
\hline Psicobiología & $\begin{array}{r}75,0 \% \\
(1,3)\end{array}$ & $27,5 \%$ & $100,0 \%$ & $\begin{array}{r}87,5 \% \\
(2,3)\end{array}$ \\
\hline Psicología Básica & $\begin{array}{r}62,1 \% \\
(1,2)\end{array}$ & $50,0 \%$ & $96,6 \%$ & $\begin{array}{r}34,5 \% \\
(-1,6)\end{array}$ \\
\hline $\begin{array}{l}\text { Psicología Evolutiva y de la } \\
\text { Educación }\end{array}$ & $\begin{array}{r}37,5 \% \\
(-2,4)\end{array}$ & $37,9 \%$ & $93,8 \%$ & $\begin{array}{r}39,6 \% \\
(-1,4)\end{array}$ \\
\hline Psicología Social & $\begin{array}{r}50,0 \% \\
(-0,2)\end{array}$ & $18,8 \%$ & $93,3 \%$ & $\begin{array}{r}33,3 \% \\
(-1,8)\end{array}$ \\
\hline
\end{tabular}

${ }^{a}$ Test de Chi cuadrado $p<0,05$. Coeficiente de contingencia, $p<0,05$

\subsection{Uso y valoración de revistas españolas}

El número total de revistas españolas valoradas ha sido de 190. En la tabla VIII se presentan aquellas que han sido valoradas por 10 o más de los encuestados.

La revista que más veces ha sido valorada, y por tanto, la más utilizada (las 3/4 partes de los encuestados), ha sido Psicothema, seguida a mucha distancia por el resto de revistas ya que son utilizadas, en el mejor de los casos, por la 1/4 parte del profesorado.

La revista con una puntuación media más alta es $\mathrm{C} \& \mathrm{E}$ : Cultura y Educación, que obtiene en todos los aspectos una puntuación de notable, seguida de la Revista Internacional de Psicología y Terapia Psicológica.

En actualidad de contenidos, todas las revistas, a excepción de Revista de Historia de la Psicología, obtienen una puntuación media superior a 6 , y muchas de ellas consiguen un notable, lo que indica que los encuestados consideran que los contenidos de las revistas son actuales.

En cuanto al rigor, las revistas con mayor puntuación son la Revista Internacional de Psicología y Terapia Psicológica $(8,2)$, Psicología Conductual $(8,0)$ y C \& E: Cultura y Educación $(8,0)$.

En presentación formal las puntuaciones obtenidas son altas y en la mayoría se consigue el notable, destacando C \& E: Cultura y Educación que alcanza casi el sobresaliente $(8,7)$. 
En calidad científica, destaca la Revista Internacional de Psicología y Terapia Psicológica con una puntuación de 8,4 , en consonancia con el alto valor obtenido también en rigor y actualidad de contenidos, siendo Papeles del Psicólogo la que menor puntuación obtiene.

Tabla VIII- Revistas españolas: Valoración media de diferentes aspectos

\begin{tabular}{|c|c|c|c|c|c|c|}
\hline & $\begin{array}{l}\mathrm{N}^{0} \text { de } \\
\text { respuestas }\end{array}$ & $\begin{array}{l}\text { Grado } \\
\text { de uso }\end{array}$ & $\begin{array}{l}\text { Actualidad } \\
\text { Contenidos }\end{array}$ & Rigor & $\begin{array}{l}\text { Present. } \\
\text { formal }\end{array}$ & $\begin{array}{l}\text { Calidad } \\
\text { científica }\end{array}$ \\
\hline Psicothema & 131 & 6,5 & 7,6 & 7,4 & 7,3 & 7,4 \\
\hline Revista de psicología general y aplicada & 47 & 4,9 & 6,4 & 6,5 & 7,5 & 6,8 \\
\hline Anales de psicología & 42 & 5,9 & 7,0 & 7,0 & 7,4 & 7,3 \\
\hline Anuario de psicología & 41 & 5,4 & 6,6 & 6,7 & 6,9 & 6,7 \\
\hline Infancia y aprendizaje & 34 & 6,6 & 7,5 & 7,5 & 7,0 & 7,5 \\
\hline Psicológica & 34 & 5,4 & 7,4 & 7,9 & 7,8 & 7,3 \\
\hline Revista de psicología social & 31 & 6,4 & 7,7 & 7,2 & 8,2 & 7,7 \\
\hline Cognitiva & 29 & 4,9 & 7,1 & 7,4 & 7,2 & 7,1 \\
\hline Análisis y modificación de conducta & 28 & 6,3 & 7,0 & 7,0 & 7,5 & 7,1 \\
\hline Psicología conductual & 26 & 7,3 & 7,9 & 8,0 & 7,1 & 7,8 \\
\hline Spanish Journal of Psychology, The & 24 & 5,0 & 7,1 & 7,3 & 7,8 & 7,2 \\
\hline Estudios de psicología & 23 & 4,9 & 7,1 & 7,1 & 7,8 & 7,0 \\
\hline Ansiedad y estrés & 22 & 5,3 & 6,6 & 6,1 & 7,8 & 6,7 \\
\hline Revista de psicología social aplicada & 20 & 6,4 & 7,3 & 7,0 & 7,8 & 7,0 \\
\hline Papeles del psicólogo & 20 & 6,1 & 6,8 & 6,0 & 6,9 & 5,9 \\
\hline Clínica y Salud & 18 & 6,1 & 6,4 & 5,9 & 6,6 & 6,1 \\
\hline Psychology in Spain & 16 & 5,8 & 7,4 & 7,6 & 7,9 & 7,8 \\
\hline Apuntes de psicología & 16 & 6,1 & 7,1 & 7,3 & 7,9 & 7,3 \\
\hline $\begin{array}{l}\text { Psiquis. Revista de psiquiatría, psicología } \\
\text { médica y psicosomática }\end{array}$ & 13 & 4,8 & 7,2 & 6,3 & 7,5 & 6,2 \\
\hline $\begin{array}{l}\text { Revista de psicología del trabajo y de las } \\
\text { organizaciones }\end{array}$ & 13 & 6,2 & 6,2 & 7,0 & 7,2 & 7,2 \\
\hline $\begin{array}{l}\text { Revista internacional de psicología y terapia } \\
\text { psicológica }\end{array}$ & 12 & 7,6 & 8,6 & 8,2 & 7,9 & 8,4 \\
\hline Boletín de psicología & 12 & 4,5 & 6,4 & 6,0 & 7,5 & 6,4 \\
\hline Cuadernos de pedagogía & 12 & 6,0 & 7,5 & 5,8 & 8,1 & 6,2 \\
\hline Revista de neurología & 11 & 6,9 & 8,2 & 6,7 & 7,6 & 7,1 \\
\hline Revista de psicología del deporte & 11 & 7,5 & 7,6 & 7,2 & 7,4 & 6,9 \\
\hline C\&E: Cultura y educación & 10 & 8,0 & 8,7 & 8,0 & 8,7 & 8,0 \\
\hline Revista de educación & 10 & 6,3 & 7,4 & 7,4 & 7,6 & 7,1 \\
\hline Revista de historia de la psicología & 10 & 6,0 & 5,5 & 6,0 & 6,2 & 6,2 \\
\hline $\begin{array}{l}\text { Revista Internacional de psicología clínica y de } \\
\text { la salud }\end{array}$ & 10 & 7,0 & 7,5 & 7,5 & 7,3 & 7,7 \\
\hline
\end{tabular}


En la tabla IX se presentan las 10 revistas más valoradas en el estudio y las 10 revistas con mayor factor de impacto en In-Recs, mostrando la correspondencia de la posición ocupada. A excepción de Psicothema, que es la revista con mayor número de respuestas y que ocupa el segundo lugar en In-Recs, y de las revistas Infancia y Aprendizaje y Psicológica, en las que existe un paralelismo en la posición en ambos lugares, en el resto de las revistas no hay relación entre la frecuencia de uso por parte de los encuestados en el estudio y su factor de impacto en In-Recs.

Tabla IX-Revistas españolas más utilizadas y su posición en In-Recs según el impacto acumulativo 2004-2008

\begin{tabular}{|l|r|r|}
\hline & $\begin{array}{l}\text { Posición } \\
\text { según el no } \\
\text { respuestas }\end{array}$ & $\begin{array}{l}\text { In-Recs } \\
\text { Posición } \\
\text { Impacto } \\
\text { 2004-2008 }\end{array}$ \\
\hline Psicothema & 1 & 2 \\
Revista de psicología general y aplicada & 2 & 19 \\
Anales de psicología & 3 & 15 \\
Anuario de psicología & 4 & 23 \\
Infancia y aprendizaje & 5 & 7 \\
Psicológica & 6 & 7 \\
Revista de psicología social & 7 & 13 \\
Cognitiva & 8 & 19 \\
Análisis y modificación de conducta & 9 & 6 \\
Psicología conductual & 10 & 19 \\
Revista internacional de psicología clínica y de la & 26 & 1 \\
salud & 35 & 3 \\
Revista de psicopatología y psicología clínica & 13 & 4 \\
Ansiedad y estrés & & 5 \\
Revista internacional de psicología y terapia & 21 & \\
psicológica & 11 & 7 \\
Spanish Journal of Psychology, The & 15 & 10 \\
Papeles del psicólogo & & 10 \\
Revista de psicología del trabajo y de las & 19 & \\
organizaciones & & \\
\hline
\end{tabular}

\subsection{Uso y valoración de revistas extranjeras}

Se han valorado un total de 358 revistas extranjeras, siendo las más frecuentes las que se muestran en la tabla $\mathrm{X}$, donde se muestran aquellas que han sido evaluadas, y por tanto utilizadas, al menos por 10 profesores e investigadores. De todas ellas, 260 sólo han sido valoradas por un encuestado, y 50 revistas por dos, lo que indica el gran 
número de revistas internacionales utilizadas en esta área de conocimiento, aunque muchas de ellas de manera muy aislada.

Entre las tres revistas más utilizadas, se encuentran Child Development, que es la que tiene mayor factor de impacto en 2008 en el ISI en la categoría de Psicología Educativa, la revista Journal of Personality and Social Psychology, con un factor de impacto que la sitúa en la posición número 3 en el área de Psicología Social y de Personalidad y en la primera posición entre las revistas experimentales de dicha área (Wikipedia contributors, 2009), y la revista Personality and Individual Differences, con un factor de impacto menor que las dos anteriores.

Hay que destacar que todas las revistas mostradas en la tabla obtienen, en general, una puntuación muy alta, en todos los aspectos evaluados, alcanzando varias de ellas el sobresaliente.

Tabla X-Revistas extranjeras: Valoración media de diferentes aspectos

\begin{tabular}{|c|c|c|c|c|c|c|}
\hline & $\begin{array}{l}\mathbf{N}^{0} \text { de } \\
\text { respuestas }\end{array}$ & $\begin{array}{l}\text { Grado } \\
\text { de uso }\end{array}$ & $\begin{array}{l}\text { Actualidad } \\
\text { Contenidos } \\
\end{array}$ & Rigor & $\begin{array}{l}\text { Present. } \\
\text { formal }\end{array}$ & $\begin{array}{l}\text { Calidad } \\
\text { científica }\end{array}$ \\
\hline Child Development & 12 & 7,7 & 8,9 & 8,7 & 8,2 & 8,7 \\
\hline $\begin{array}{l}\text { Journal of Personality and } \\
\text { Social Psychology }\end{array}$ & 12 & 8,2 & 9,3 & 9,2 & 6,8 & 9,2 \\
\hline $\begin{array}{l}\text { Personality and Individual } \\
\text { Differences }\end{array}$ & 12 & 8,2 & 8,2 & 8,6 & 8,5 & 8,3 \\
\hline Psychological Review & 10 & 7,2 & 8,5 & 8,8 & 7,7 & 8,8 \\
\hline $\begin{array}{l}\text { Behavior Research and } \\
\text { Therapy }\end{array}$ & 8 & 8,0 & 9,1 & 8,9 & 7,9 & 9,1 \\
\hline Developmental Psychology & 8 & 7,9 & 9,2 & 9,2 & 9,1 & 9,0 \\
\hline $\begin{array}{l}\text { Journal of Applied } \\
\text { Psychology }\end{array}$ & 8 & 7,7 & 8,9 & 8,9 & 8,6 & 9,4 \\
\hline $\begin{array}{l}\text { European Journal of } \\
\text { Psychological Assessment }\end{array}$ & 7 & 7,6 & 8,3 & 8,1 & 8,2 & 7,9 \\
\hline $\begin{array}{l}\text { Educational and } \\
\text { Psychological } \\
\text { Measurement }\end{array}$ & 6 & 8,9 & 8,7 & 8,7 & 8,2 & 9,0 \\
\hline Memory and Cognition & 6 & 8,0 & 9,2 & 8,7 & 8,3 & 8,5 \\
\hline Psychological Methods & 6 & 9,2 & 9,7 & 9,5 & 7,5 & 8,8 \\
\hline Health Psychology & 5 & 7,2 & 9,4 & 9,6 & 8,0 & 9,6 \\
\hline $\begin{array}{l}\text { Journal of Consulting and } \\
\text { Clinical Psychology }\end{array}$ & 5 & 8,2 & 9,4 & 9,2 & 7,5 & 9,4 \\
\hline $\begin{array}{l}\text { Journal of Educational } \\
\text { Psychology }\end{array}$ & 5 & 8,0 & 8,8 & 8,8 & 8,5 & 9,2 \\
\hline $\begin{array}{l}\text { Journal of Experimental } \\
\text { Psychology }\end{array}$ & 5 & 7,4 & 8,6 & 9,4 & & 9,6 \\
\hline $\begin{array}{l}\text { Revista Latinoamericana de } \\
\text { Psicología }\end{array}$ & 5 & 7,8 & 7,8 & 7,0 & 7,4 & 7,4 \\
\hline
\end{tabular}




\begin{tabular}{|l|r|r|r|r|r|r|}
\hline Applied Psyholinguistic & 4 & 8,2 & 8,5 & 9,2 & 8,8 & 9,0 \\
Applied Psychological & & & & & & \\
Measurement & 4 & 8,2 & 9,2 & 9,2 & 8,3 & 9,2 \\
Applied Psychology: An & 4 & 8,2 & 8,7 & 8,5 & 8,8 & 8,7 \\
International Review & & & & & & \\
British Journal of & 4 & 7,2 & 8,7 & 8,2 & 8,8 & 8,0 \\
Educational Psychology & & & & & & \\
European Journal of & 4 & 7,2 & 7,2 & 7,2 & 8,0 & 7,5 \\
Personality & 4 & 8,5 & 8,2 & 7,7 & 8,8 & 7,5 \\
Journal of Adolescence & & & & & & \\
Journal of Exp. Psychol: & 4 & 8,5 & 10 & 9,0 & 9,3 & 9,0 \\
Learning, Memory and & 4 & 8,2 & 9,0 & 8,7 & 9,0 & 8,7 \\
Cognition & 4 & 8,0 & 9,7 & 8,0 & 9,0 & 9,0 \\
Neuroimage & 4 & 8,2 & 10 & 10 & 8,5 & 9,0 \\
Neuroreport & 4 & 7,5 & 9,5 & 9,0 & 9,0 & 8,7 \\
Psychological Bulletin & 4 & 7,7 & 9,5 & 9,7 & 6,3 & 9,5 \\
\hline Psychological Reports & & & & & \\
Psychometrika & & & & & \\
\hline
\end{tabular}

\subsection{Uso de revistas por áreas de conocimiento}

En las tablas XI a la XVI se presentan las revistas españolas y extranjeras más utilizadas por los profesores e investigadores en cada una de las áreas de conocimiento. Un alto porcentaje de uso en el área para una revista significa que es utilizada por un alto número de profesores que pertenecen a dicha área, mientras que un bajo porcentaje significa lo contrario. Un alto porcentaje de uso en otras áreas significa que un alto número de profesores de otras áreas utilizan la revista.

El análisis de los dos porcentajes nos puede indicar la inter o multidisciplinariedad o la especialización de las revistas. Se pueden dar cuatro situaciones:

- Revistas con altos porcentajes de uso en el área y en otras áreas, nos está indicando que son utilizadas de manera frecuente por diferentes áreas de conocimiento y que, lógicamente, tratan temas inter o multidisciplinares.

- Revistas con altos porcentajes de uso en el área y bajos porcentajes de uso en otras áreas, nos indica que sólo resultan de utilidad en esa área de conocimiento y que, por tanto, están especializadas en dicha área.

- Revistas con bajos porcentajes de uso en el área y altos en otras áreas, indica que esas revistas son utilizadas ocasionalmente en el área de conocimiento específica mientras que pueden estar especializadas en otra área o ser multidisciplinares, pero en otras áreas de conocimiento.

- Revistas con bajos porcentajes de uso tanto en el área específica como en otras, revela que se utilizan de manera ocasional y puntual por los profesores e investigadores de Psicología. 
Al comparar el rango de las revistas españolas más veces valoradas con el conseguido en el estudio realizado por Alcaín y Román (2005), se observa que en el área de Metodología de las Ciencias del Comportamiento existe una coincidencia plena; en el área de Personalidad, Evaluación y Tratamiento Psicológico, sólo la revista Clínica y Salud no aparece entre las más valoradas en el estudio de Alcaín; en el área de Psicología Básica, coinciden las 4 primeras, y Psicológica aparece en octavo lugar; en el área de Psicología Evolutiva y de la Educación, las revistas Anales de Psicología y Cuadernos de Pedagogía no aparecen en el rango de Alcaín; en el área de Psicología Social, aparecen todas las revistas, si bien el rango cambia en alguna de ellas. 
Tabla XI-Revistas más utilizadas en el área de Metodología de las Ciencias del Comportamiento

\begin{tabular}{|l|l|r|r|r|}
\hline \multicolumn{2}{|l|}{} & $N^{\mathbf{a}}$ & \multicolumn{1}{l}{$\begin{array}{l}\text { \% uso en } \\
\text { el área }\end{array}$} & $\begin{array}{l}\text { \% uso en } \\
\text { otras áreas }\end{array}$ \\
\hline \multicolumn{2}{|l|}{ Revistas españolas } & & & \\
\hline 1 & Psicothema & 19 & $100 \%$ & $85,5 \%$ \\
2 & Psicológica & 7 & $84,2 \%$ & $52,9 \%$ \\
3 & Metodología de las Ciencias del & 5 & $26,3 \%$ & $10,5 \%$ \\
& Comportamiento & 5 & $26,3 \%$ & $0 \%, 1 \%$ \\
4 & Anuario de Psicología & & & \\
5 & Metodología de Encuestas & & & \\
\hline Revistas extranjeras & 6 & $31,6 \%$ & $0 \%$ \\
\hline 1 & Educational and Psychological & 6 & $31,6 \%$ & $0 \%$ \\
& Measurement & 4 & $21,0 \%$ & $0 \%$ \\
2 & Psychological Methods & 4 & $21,0 \%$ & $0 \%$ \\
\hline 3 & Applied Psychological Measurement & & \\
4 & Psychometrika & & \\
\hline
\end{tabular}

Tabla XII- Revistas más utilizadas en el área de Personalidad, Evaluación y Tratamiento Psicológico

\begin{tabular}{|l|l|r|r|r|}
\hline & \multicolumn{1}{|l|}{$\begin{array}{l}\text { Na uso en } \\
\text { el área }\end{array}$} & $\begin{array}{l}\text { \% uso en } \\
\text { otras áreas }\end{array}$ \\
\hline \multicolumn{2}{|l|}{ Revistas españolas } & & & \\
\hline 1 & Psicothema & 34 & $82,9 \%$ & $74,0 \%$ \\
2 & Análisis y modificación de conducta & 21 & $51,2 \%$ & $25,0 \%$ \\
3 & Psicología conductual & 21 & $51,2 \%$ & $19,2 \%$ \\
4 & Clínica y Salud & 16 & $39,0 \%$ & $11,1 \%$ \\
5 & Ansiedad y Estrés & & $24,4 \%$ & $54,5 \%$ \\
\hline Revistas extranjeras & 9 & $21,9 \%$ & $25,0 \%$ \\
\hline 1 & Personality and Individual Differences & 8 & $19,5 \%$ & $0 \%$ \\
2 & Behavior Research and Therapy & 4 & $9,7 \%$ & $0 \%$ \\
3 & European Journal of Personality & 4 & $9,7 \%$ & $20,0 \%$ \\
4 & Health Psychology & 4 & $9,7 \%$ & $20,0 \%$ \\
5 & Journal of Consulting and Clinical & & & \\
& Psychology & 4 & $9,7 \%$ & $66,6 \%$ \\
\hline 5 & Journal of Personality and Social & & & \\
\end{tabular}


Tabla XIII-Revistas más utilizadas en el área de Psicobiología

\begin{tabular}{|l|l|r|r|r|}
\hline & $N^{\mathbf{a}}$ & $\begin{array}{l}\% \text { uso en } \\
\text { el área }\end{array}$ & $\begin{array}{l}\% \text { uso en } \\
\text { otras } \\
\text { áreas }\end{array}$ \\
\hline \multicolumn{2}{|l|}{ Revistas españolas } & & & \\
\hline 1 & Revista de Neurología & 6 & $75,0 \%$ & $45,4 \%$ \\
2 & Psicothema & 5 & $62,5 \%$ & $96,2 \%$ \\
3 & Ansiedad y Estrés & 4 & $50,0 \%$ & $81,8 \%$ \\
4 & Cognitiva & 4 & $50,0 \%$ & $86,2 \%$ \\
\hline Revistas extranjeras & & & \\
\hline 1 & Neuroreport & 4 & $50,0 \%$ & $0 \%$ \\
\hline
\end{tabular}

Tabla XIV-Revistas más utilizadas en el área de Psicología Básica

\begin{tabular}{|c|c|c|c|c|}
\hline & & $\mathbf{N}^{\mathbf{a}}$ & $\begin{array}{l}\text { \% uso en el } \\
\text { área }\end{array}$ & $\begin{array}{l}\text { \% uso en } \\
\text { otras áreas }\end{array}$ \\
\hline \multicolumn{5}{|c|}{ Revistas españolas } \\
\hline 1 & Psicothema & 20 & $66,6 \%$ & $84,7 \%$ \\
\hline 2 & Cognitiva & 16 & $53,3 \%$ & $44,8 \%$ \\
\hline 3 & Revista de Psicología General y Aplicada & 15 & $50,0 \%$ & $68,1 \%$ \\
\hline 4 & Estudios de Psicología & 13 & $43,3 \%$ & $43,5 \%$ \\
\hline 5 & Psicológica & 13 & $43,3 \%$ & $61,7 \%$ \\
\hline \multicolumn{5}{|c|}{ Revistas extranjeras } \\
\hline 1 & Psychological Review & 6 & $20,0 \%$ & $40,0 \%$ \\
\hline 2 & Journal of Experimental Psychology & 5 & $16,6 \%$ & $0 \%$ \\
\hline 3 & Memory and Cognition & 5 & $16,6 \%$ & $16,6 \%$ \\
\hline 4 & $\begin{array}{l}\text { Journal of Exp. Psychol: Learnign, } \\
\text { Memory and Cognition }\end{array}$ & 4 & $13,3 \%$ & $0 \%$ \\
\hline
\end{tabular}


Tabla XIV-Revistas más utilizadas en el área de Psicología Evolutiva y de la Educación

\begin{tabular}{|l|l|r|r|r|}
\hline \multicolumn{2}{|l|}{} & $\mathbf{N}^{\mathbf{a}} \begin{array}{l}\% \\
\text { en el uso } \\
\text { área }\end{array}$ & $\begin{array}{l}\text { \% uso } \\
\text { en otras } \\
\text { áreas }\end{array}$ \\
\hline \multicolumn{2}{|c|}{ Revistas españolas } & & & \\
\hline 1 & Psicothema & 30 & $62,5 \%$ & $77,1 \%$ \\
2 & Infancia y Aprendizaje & 27 & $56,2 \%$ & $20,6 \%$ \\
3 & Anales de Psicología & 14 & $29,2 \%$ & $66,6 \%$ \\
4 & Anuario de Psicología & 13 & $27,1 \%$ & $68,3 \%$ \\
5 & Cuadernos de Pedagogía & 11 & $22,9 \%$ & $8,3 \%$ \\
\hline Revistas extranjeras & & & \\
\hline 1 & Child Development & 10 & $20,8 \%$ & $16,6 \%$ \\
2 & Developmental Psychology & 8 & $16,6 \%$ & $0 \%$ \\
3 & Journal of Educational Psychology & 5 & $10,4 \%$ & $0 \%$ \\
\hline
\end{tabular}

Tabla XVI- Revistas más utilizadas en el área de Psicología Social

\begin{tabular}{|l|l|r|r|r|}
\hline \multicolumn{2}{|l|}{} & \multicolumn{1}{|c|}{$\begin{array}{l}\text { Na uso } \\
\text { en el } \\
\text { área }\end{array}$} & $\begin{array}{l}\% \\
\text { en otras } \\
\text { áreas }\end{array}$ \\
\hline \multicolumn{2}{|l|}{ Revistas españolas } & & & \\
\hline 1 & Revista de Psicología Social & 23 & $76,6 \%$ & $34,8 \%$ \\
2 & Psicothema & 22 & $73,3 \%$ & $83,2 \%$ \\
3 & Revista de Psicología Social Aplicada & 17 & $56,6 \%$ & $15,0 \%$ \\
4 & Revista de Psicología del Trabajo y de las & 9 & $30,0 \%$ & \\
& Organizaciones & 8 & $26,6 \%$ & $80,7 \%$ \\
5 & Anuario de Psicología & 8 & $26,6 \%$ & $83,0 \%$ \\
\hline 5 & Revista de Psicología General y Aplicada & & & \\
\hline Revistas extranjeras & 4 & $13,3 \%$ & $66,6 \%$ \\
\hline 1 & Journal of Personality and Social Psychology & 3 & $10,0 \%$ & $25,0 \%$ \\
2 & Applied Psychology: An International Review & 3 & $10,0 \%$ & $0 \%$ \\
\hline 3 & European Journal of Social Psychology & 3 & $10,0 \%$ & $0 \%$ \\
4 & Human Relations & 3 & $10,0 \%$ & $62,5 \%$ \\
\hline 5 & Journal of Applied Psychology & 3 & $10,0 \%$ & $0 \%$ \\
6 & Journal of Environmental Psychology & 3 & $10,0 \%$ & $0 \%$ \\
\hline 7 & Journal of Organizational Behavior & &
\end{tabular}




\section{6. Comparación de la valoración de revistas españolas y revistas extranjeras}

En la tabla XVII se comparan las puntuaciones finales medias obtenidas, junto con sus intervalos de confianza al 95\%, en cada uno de los aspectos por las revistas nacionales y extranjeras.

El aspecto mejor valorado en las revistas nacionales es la presentación formal, y el peor el rigor de los temas tratados en los artículos. En las revistas extranjeras la puntuación más alta la obtiene la actualidad de contenidos, si bien todos los aspectos valorados consiguen un notable alto.

Tabla XVII- Valoración media global de revistas españolas y revistas extranjeras

\begin{tabular}{|l|l|l|}
\hline & $\begin{array}{l}\text { Revistas } \\
\text { nacionales }\end{array}$ & $\begin{array}{l}\text { Revistas } \\
\text { extranjeras }\end{array}$ \\
\hline Grado de uso & $6,0(5,8-6,1)$ & $7,7(7,6-7,9)$ \\
Actualidad de contenidos & $7,1(6,9-7,2)$ & $8,7(8,6-8,8)$ \\
Rigor & $6,9(6,8-7,0)$ & $8,5(8,4-8,6)$ \\
\hline Presentación formal & $7,4(7,3-7,5)$ & $8,3(8,2-8,4)$ \\
Calidad científica & $7,0(6,8-7,1)$ & $8,6(8,5-8,7)$ \\
\hline
\end{tabular}

A la hora de comparar las revistas nacionales y extranjeras, podemos observar que las revistas extranjeras obtienen una puntuación más alta en todos los aspectos valorados, lo que coincide con los resultados de Alcaín y Román (2005) donde los profesores consideran mejores las revistas extranjeras que las españolas.

\section{CONCLUSIONES}

El estudio ha permitido conocer la opinión de un conjunto de profesores universitarios pertenecientes al área de Psicología en relación a diversos aspectos relacionados con el uso y evaluación de revistas científicas. Al ser un estudio exploratorio y, dado que se desconoce la representatividad de la muestra, las conclusiones de este estudio se presentan a continuación como hipótesis científicas a contrastar en futuros trabajos:

- El tema tratado es el principal criterio utilizado en la selección de un artículo.

- Los catedráticos de universidad conceden más importancia al autor de una publicación y menos a la fecha de publicación que el resto de categorías profesionales.

- La revisión anónima por expertos sigue siendo considerado el sistema de selección de artículos más adecuado en la actualidad.

- Publicar en una revista extranjera no es garantía de mayor calidad que publicar en una revista nacional. 
- Para los asociados y titulares y catedráticos de escuela universitaria la evaluación de los medios de publicación no es el medio más adecuado para evaluar la investigación de un autor.

- Los titulares y catedráticos de escuela universitaria creen que la calidad de las revistas no depende del número de citas que reciben sus artículos.

- La revista nacional más utilizada por el personal docente e investigador en nuestro país en todas las áreas de conocimiento es Psicothema, con una valoración de notable en los aspectos evaluados: actualidad de contenidos, rigor, presentación formal y calidad científica global.

- En el ámbito internacional, ninguna revista destaca por su alto grado de uso ya que son muchas las revistas utilizadas, aunque por un número bajo de profesores.

- Las revistas extranjeras obtienen más puntuación en todos los aspectos que las revistas nacionales.

\section{REFERENCIAS BIBLIOGRÁFICAS}

AGUDELO, D., BRETÓN-LÓPEZ, J. y BUELA-CASAL, G. (2003). "Análisis bibliométrico de las revistas de Psicología Clínica editadas en castellano". Psicothema 15 (4), pp. (507-516).

ALCAÍN PARTEARROYO, M.D. y ROMÁN ROMÁN, A. (2005a). "Hacia una valoración integrada de las revistas españolas de Ciencias Sociales y Humanas: las revistas de Psicología". Psicothema 17 (2), pp. (179-189). ---. (2005b). "Respuesta a las consideraciones realizadas por Carretero y colaboradores al trabajo titulado "Hacia una valoración integrada de las revistas españolas de Ciencias Sociales y Humanas: las revistas de Psicología”. Psicothema 17 (4), pp. (676-678).

ALCAÍN PARTEARROYO, M.D. y RUIZ-GALVEZ, M. (2001). "Evaluación de revistas científicas españolas de Psicología". Revista de Historia de la Psicología 22 (1), pp. 25-40.

ALEXANDER, J.C. y MABRY, R.H. (1994). "Relative significance of journals, authors and articles cited in financial research". Journal of Finance 49 (2), pp. (697712).

BAKER, D.R. (1992). "A structural analysis of the social work journal network: 19851986.". Journal of Social Service Research 15 (3-4), pp. (153-168).

BEATTIE, V. y GOODACRE, A. (2005). A new method for ranking academic journals in accounting and finance. University of Sterling Accounting, Finance and Law.

BOOR, M. (1973). "Unfamiliarity breeds disdain: comment on department chairmen's ratings of psychology journals". American Psychologist 28 , pp. (1012-1013).

BRINN, T.; JONES, M.J. y PENDLEBURY, M. (1996). "UK accountants' perceptions of research journal quality". Accounting and Business Research 26 (3), pp. (265278).

BUELA-CASAL, G. (2005). "La evaluación de la calidad en el proceso de convergencia europea”. Revista Mexicana de Psicología 22, pp. (306-314). 
BUELA CASAL, G. (2003). "Evaluación de la calidad de los artículos y de las revistas científicas: propuesta del factor de impacto ponderado y de un índice de calidad". Psicothema 15 (1), pp. (23-25).

BUELA-CASAL, G., CARRETERO-DIOS, H. y DE LOS SANTOS-ROIG, M. (2002). "Estudio comparativo de las revistas de Psicología en castellano con factor de impacto". Psicothema 14 (4), pp. (837-852).

BUSH, I.R.; EPSTEIN, I. y SAINZ, A. (1997). "The use of social science sources in social work practice journals: an application of citation analysis". Social Work Research 21 (1), pp. (45-56).

CARPINTERO, H., GOTOR, A. y MIRALLES, J.L. (1983a). "El tipo de revista y su influencia en la colaboración, las materias y la productividad de la revista". Revista de Historia de la Psicología 4 (4), pp. 377-389.

CARPINTERO, H., GOTOR, A. y MIRALLES, J.L. (1983b). "Los editores de revistas científicas de Psicología". Revista de Psicología General y Aplicada 38 (2), pp. 317 348.

CARPINTERO, H. y TORTOSA, F. (1996). "La Psicología española a través de la Revista de Psicología General y Aplicada". Revista de Psicología General y Aplicada 49 (3-4), pp. 373-410.

CARRETERO-DIOS, H.; DE LOS SANTOS ROIG, M. y BUELA CASAL, G. (2005). "Evaluación de la calidad de las revistas científicas de Psicología publicadas en España. Consideraciones al trabajo de Alcaín y Román, 2005”. Psicothema 17 (4), pp. (669-675).

CHAN, K.C.; FOK, R.C.W. y PAN, M.S. (2000). "Citation-based finance journal rankings: an update". Financial Practice and Education 10 (1), pp. (132-141).

CHEUNG, K.M. (1990). "Interdisciplinary relationships between social work and other disciplines: a citation study". Social Work Research and Abstracts 26 (3), pp. (2329).

CNAAN, R.A.; CAPUTO, R.K. y SHMUELY, Y. (1994). "Senior faculty perceptions of social work journals". Journal of Social Work Education (30), pp. (185-199).

Council on Social Work Education (2002). Alexandria, VA: Author.

DORELAN, P. (1989). "On the ranking of psychological journals". Information Processing and Management (25), pp. (205-214).

EASTON, G. y EASTON, D.M. (2003). "Marketing journals and the research assessment exercise". Journal of Marketing Management 19 (1-2), pp. (5-24).

FIGUEREDO, E. (2006). "The numerical equivalence between the impact factor of journals and the quality of the articles". Journal of the American Society for Information Science and Technology 57 (11), pp. (1561).

FRANKE, R.H.; EDLUND, T.W. y OSTER, F. (1990). "The development of strategic management: journal quality and article impact". Strategic Management Journal (11), pp. (243-253).

FRY, E.H.; WALTERS, C.G. y SCHEUERMANN, L.E. (1985). "Perceived quality of fifty selected journals: academicians and practitioners". Academy of Marketing Science (13), pp. (352-361). 
FURR, A. (1995). "The relative influence of social work journals: impact factors $v s$. core influence". Journal of Social Work Education (31), pp. (38-46).

GARAND, J.C. (1990). "An alternative interpretation of recent political science journal evaluations". Political Science and Politics (23), pp. (448-451).

GEARY, J.; MARRIOTT, L. y ROWLINSON, M. (2004). "Journal rankings in business and management and the 2001 research assessment exercise in the UK". British Journal of Management 15 (2), pp. (95-141).

GILES, M.W.; MIZELL, F. y PATTERSON, D. (1989). "Political scientists' journal evaluations revisited". Political Science and Politics (22), pp. (613-617).

GLENN, N.D. (1971). "American sociologist's evaluations of sixty-three journals". The American Sociologist (6), pp. (298-303).

GOTOR, A. (1985) y MIRALLES, J.L. "Dispersión, constancia y permanencia de citación: un índice ponderado de visibilidad". Revista de Historia de la Psicología, 6 (3), 267-281.

GRUPO DE INVESTIGACIÓN ERCE. "ERCE. Evaluación de las revistas españolas de Humanidades $y$ Ciencias Sociales [en línea]." Grupo de investigación ERCE. 2007. <http://erce.unizar.es> [última consulta 25-10-2009].

GYNTHER, M.D. (1973). "On Mace and Warner's journal ratings". American Psychologist (28), pp. (1013).

HAYSLETT, M.M. y WILDEMUTH, B.M. (2004). "Pixels or pencils? The relative effectiveness of web-based versus paper surveys". Library and Information Science Research 26 , pp. (73-93).

HERRON, T.L. y HALL, T.W. (2005). "Faculty perceptions of journals: quality and publishing feasibility". Journal of Accounting Education 22 , pp. (175-210).

LAMARCA LANGA, G.; ARQUERO AVILES, R.; ESTEBAN NAVARRO, M.A.; MORENO VERNIS, M.; SALVADOR OLIVÁN, J.A.; GORDILLO ACOSTA, I. y NOVALES ARALUCE, A. (2005). "Evaluación de la calidad de las revistas científicas españolas en Humanidades y Ciencias Sociales". Boletín de ANABAD 55 (12), pp. (377-391).

LESTER, J.P. (1990). "Evaluating the evaluators: accrediting knowledge and the ranking of political science journals". Political Science and Politics (23), pp. (445-447).

LINDSEY, D. (1978). "The operation of professional journals in social work". Journal of Sociology and Social Work (17), pp. (273-298).

LINDSEY, D. y KIRK, S. (1992). "The role of social work journals in the development of a knowledge base for the profession". Social Service Review (66), pp. (295-310).

LOWE, A. y LOCKE, J. (2005). "Perceptions of journal quality and research paradigm: results of a web-based survey of British accounting academics". Accounting, Organizations and Society 30, pp. (81-98).

MACE, K.C. y WARNER, H.D. (1973). "Ratings of psychology journals". American Psychologist (28), pp. (184-186).

McROBERTS, M.H. y McROBERTS, B.R. (1989). "Problems of citation analysis: a critical review". Journal of the American Society for Information Science and Technology 40 (5), pp. (342-349). 
MILLER, J.; DALY, J.; WOOD, M.B., A. y ROPER, M. (1996). "Electronic bulleting board distributed questionnaires for exploratory research". Journal of the American Society for Information Science and Technology 22 (2), pp. (107-115).

MILLER, J.; DALY, J.; WOOD, M.B., A. y ROPER, M. (1996). "Electronic bulleting board distributed questionnaires for exploratory research". Journal of the American Society for Information Science and Technology 22 (2), pp. (107-115).

NELSON, T.M.; BUSS, A.R. y KATZKO, M. (1983). "Rating of scholarly journals by chairpersons in the social sciences". Research in Higher Education 19 (4), pp. (469497).

NISONGER, T.E. (1993). "A ranking of political science journals based on citation data". Serials Review 19 (4), pp. (7-14).

OTLEY, D. (2002). "British research in accounting and finance (1996-2000): the 2001 research assessment exercise". British Accounting Review 34 (4), pp. (387-417).

PARAMESWARAN, A. y SEBASTIAN, R. (2006). "The value of South and Southeast Asian studies journal rankings". Serials Review 32 (3), pp. (154-163).

PARKER, L.; GUTHRIE, J. y GRAY, R. (1998). "Accounting and management research: passwords from the gatekeepers". Accounting, Auditing and Accountability Journal 11 (4), pp. (371-402).

PEÑARANDA ORTEGA, M., QUIÑONES VIDAL, E., LÓPEZ GARCÍA, J.J. (2005). "Veinte años de Anales de Psicología: Una revista con raíces académicas (19842004)". Anales de Psicología 21 (2), pp. 181-198.

PÉREZ ÁLVAREZ-OSSORIO, J.R. (2001). "La evaluación de las revistas científicas". Bid: Biblioteconomía y Documentación [en línea]. $<$ http://www.ub.es/bid/06perez2.htm> [última consulta 26-10-2009].

REAMER, F.G. (1992). "Publishing and perishing in social work education". Journal of Social Work Education (28), pp. (129-131).

SALVADOR OLIVÁN, J.A.; LAMARCA LANGA, G. y ARQUERO AVILÉS, R. (2009a). "La percepción de la calidad y la utilización de revistas académicas por el personal docente e investigador del área de conocimiento de Biblioteconomía y Documentación". Documentación de las Ciencias de la Información 32, pp. (193-205).

SALVADOR OLIVÁN, J.A.; LAMARCA LANGA, G. y ARQUERO AVILÉS, R. (2009b). "Los profesores e investigadores universitarios de Ciencias Sociales y Humanidades y los artículos académicos: criterios de selección y opinión". Revista General de Información y Documentación 19 (1), pp. (173-188).

TEEVAN, J.J. (1980). "Journal prestige and quality of sociological articles". The American Sociologist (15), pp. (109-112).

TORTOSA GIL, F. Y CIVERA MOYÁ, C. (2001). "Revistas y disciplina psicológica. Cien años de encuentro". Papeles del Psicólogo (79), pp. 3-14.

WEST, C.K.; CARMODY, C. y STALLINGS, W.M. (1983). "The quality of research articles in the journal of educational research". Journal of Education Research 77 (2), pp. (70-76).

WIKIPEDIA CONTRIBUTORS. Journal of Personality and Social Psychology. Wikipedia, The Free Encyclopedia. September 29, 2009, 21: 21UTC. Disponible en: 